\title{
Boundaries of Disk-Like Self-affine Tiles
}

\author{
King-Shun Leung • Jun Jason Luo
}

Received: 3 June 2012 / Revised: 12 March 2013 / Accepted: 22 April 2013 /

Published online: 15 May 2013

(C) Springer Science+Business Media New York 2013

\begin{abstract}
Let $T:=T(A, \mathcal{D})$ be a disk-like self-affine tile generated by an integral expanding matrix $A$ and a consecutive collinear digit set $\mathcal{D}$, and let $f(x)=x^{2}+p x+q$ be the characteristic polynomial of $A$. In the paper, we identify the boundary $\partial T$ with a sofic system by constructing a neighbor graph and derive equivalent conditions for the pair $(A, \mathcal{D})$ to be a number system. Moreover, by using the graph-directed construction and a device of pseudo-norm $\omega$, we find the generalized Hausdorff dimension $\operatorname{dim}_{H}^{\omega}(\partial T)=2 \log \rho(M) / \log |q|$ where $\rho(M)$ is the spectral radius of certain contact matrix $M$. Especially, when $A$ is a similarity, we obtain the standard Hausdorff dimension $\operatorname{dim}_{H}(\partial T)=2 \log \rho / \log |q|$ where $\rho$ is the largest positive zero of the cubic polynomial $x^{3}-(|p|-1) x^{2}-(|q|-|p|) x-|q|$, which is simpler than the known result.
\end{abstract}

Keywords Boundary $\cdot$ Self-affine tile $\cdot$ Sofic system $\cdot$ Number system $\cdot$ Neighbor graph $\cdot$ Contact matrix $\cdot$ Graph-directed set $\cdot$ Hausdorff dimension

\section{Introduction}

Let $M_{n}(\mathbb{Z})$ denote the set of $n \times n$ matrices with entries in $\mathbb{Z}$ and let $A \in M_{n}(\mathbb{Z})$ be expanding (i.e., all eigenvalues of $A$ have moduli $>1$ ). Assume $|\operatorname{det}(A)|=|q|$, and $\mathcal{D}=\left\{0, d_{1}, \ldots, d_{|q|-1}\right\} \subset \mathbb{Z}^{n}$ with $|q|$ distinct vectors. We call $\mathcal{D}$ a digit set

\footnotetext{
K.-S. Leung

Department of Mathematics and Information Technology, The Hong Kong Institute of Education, Hong Kong, Hong Kong

e-mail: ksleung@ied.edu.hk

J. J. Luo $(\varangle)$

Department of Mathematics, Shantou University, Shantou 515063, Guangdong, China

e-mail: luojun2011@yahoo.com.cn
} 
and $(A, \mathcal{D})$ a self-affine pair. It is well known that there exists a unique self-affine set $T:=T(A, \mathcal{D})[20]$ satisfying

$$
T=A^{-1}(T+\mathcal{D})=\left\{\sum_{i=1}^{\infty} A^{-i} d_{j_{i}}: d_{j_{i}} \in \mathcal{D}\right\}
$$

If $T$ has non-void interior, then there exists a subset $\mathcal{J} \subset \mathbb{Z}^{n}$ such that

$$
T+\mathcal{J}=\mathbb{R}^{n} \text { and }(T+t)^{\circ} \cap\left(T+t^{\prime}\right)^{\circ}=\emptyset, \quad t \neq t^{\prime}, t, t^{\prime} \in \mathcal{J},
$$

thus $T$ is called a self-affine tile and $\mathcal{J}$ a tiling set. $T+\mathcal{J}$ is called a tiling of $\mathbb{R}^{n}$, and a lattice tiling if $\mathcal{J}$ is a lattice [22].

The topological properties of self-affine tiles and their boundaries, such as connectedness, local connectedness, or disk-likeness (i.e., homeomorphic to the closed unit disk), have attracted a lot of interest. A systematical study on the connectedness of self-affine tiles was due to Kirat and Lau [19], they mainly concerned a class of tiles $T(A, \mathcal{D})$ generated by the consecutive collinear $(\mathrm{CC})$ digit sets $\mathcal{D}:=\mathcal{D}(v,|q|)=\{0,1, \ldots,|q|-1\} v, v \in \mathbb{Z}^{n} \backslash\{0\}$ via the algebraic property of the characteristic polynomial of the matrix $A$. More general cases on non-consecutive collinear or non-linear digit sets were considered by $[6,18,25]$.

The question of disk-likeness was first investigated by Bandt and Gelbrich [3] for self-affine tiles in $\mathbb{R}^{2}$ with $|\operatorname{det}(A)|=2$ or 3 . They observed that the characteristic polynomial of $A \in M_{2}(\mathbb{Z})$ is of the form:

$$
f(x)=x^{2}+p x+q, \text { with }|p| \leq q, \text { if } q \geq 2 ; \quad|p| \leq|q+2|, \text { if } q \leq-2 .
$$

By studying the neighborhood structure of $T$, Bandt and Wang [5] proved that a tile $T$ with no more than six neighbors is disk-like if and only if $T$ is connected. A translation of the tile $T+\ell, \ell \in \mathcal{J}$ is called a neighbor of $T$ if $T \cap(T+\ell) \neq \emptyset$. Making use of this criterion, Leung and Lau [24] then gave a complete characterization of the disk-likeness of self-affine tiles with CC digit sets. Gmainer and Thuswaldner [14] considered the disk-likeness of tiles with non-collinear digit sets arising from polyominoes, and Kirat [18] proposed necessary and sufficient conditions for such tiles to be disk-like in general. By using the neighbor map technique, Bandt and Mesing [4] constructed a kind of finite type self-affine tiles and discussed their disk-likeness as well.

Theorem 1.1 ([24]) Let $A \in M_{2}(\mathbb{Z})$ be an expanding matrix with characteristic polynomial $f(x)=x^{2}+p x+q$. Then for any $C C$ digit set $\mathcal{D}(v,|q|)$ in $\mathbb{Z}^{2}$ such that $v$, A $v$ are linearly independent, $T$ is a disk-like tile if and only if $2|p| \leq|q+2|$.

Moreover, when $p=0, T$ is a square tile; when $p \neq 0, T$ is a hexagonal tile.

The boundary of a self-affine tile has more complicated geometric structure than the tile itself, hence it is also of considerable interest. The dimension of the boundary of a self-similar tile (where the expanding matrix $A$ is a similarity) has been studied extensively in the literature. Strichartz and Wang [31] described the boundary set as a 
graph-directed set and gave an algorithm for finding the dimension of the boundary, various other methods can be founded in [8, 17,23,32].

Recently, Akiyama and Loridant [1,2] provided a new method to parameterize the boundary set and reproved Theorem 1.1 by showing that the boundary of $T$ is a simple closed curve. In the present paper, we go further to explore the structure of the boundary of the $T$ defined in Theorem 1.1. For convenience, we call such $T$ a $C C$ tile. If it is also disk-like, we call it a disk-like CC tile.

First we establish a neighbor graph of $T$ such that the boundary $\partial T$ is identified as the union of all one-sided infinite paths of this graph. Hence $\partial T$ determines a sofic system [11]. The neighbor graph technique is classical in the study of tiling theory $[3,4]$. However, it will be shown that we use the technique here from a different aspect. As self-affine tiles can be studied in the context of number systems [29], it is worth studying the conditions for the self-affine pair $(A, \mathcal{D})$ to be a number system. We give the answer when $T(A, \mathcal{D})$ is disk-like.

Theorem 1.2 Let $T=T(A, \mathcal{D})$ be a disk-like CC tile. Then the following are equivalent:

(i) $(A, \mathcal{D})$ is a number system.

(ii) $0 \in T^{\circ}$.

(iii) $f(x)=x^{2}+p x+q$ with $-1 \leq p$ and $q \geq 2$.

(iv) For all neighbors $T+\ell, \ell=\sum_{i=0}^{k} a_{i} A^{i} v \in \mathcal{D}_{A, k+1}$ for some $k \in \mathbb{Z}$ with $a_{k}=1$ and $a_{i} \in D$ where $0 \leq i<k$.

In [31], Strichartz and Wang applied the graph-directed iterated function system (GIFS) to represent the boundary of a self-affine tile, but they were not sure whether the GIFS satisfies the open set condition or not. Our second aim is to give a positive answer for the disk-like CC tile and estimate the generalized Hausdorff dimension ( $\operatorname{dim}_{H}^{\omega}$ ) of the boundary by using a pseudo-norm $\omega[16,26]$ instead of Euclidean norm.

Theorem 1.3 The generalized Hausdorff dimension of the boundary of disk-like CC tile $T$ is

$$
\operatorname{dim}_{H}^{\omega}(\partial T)=\frac{2 \log \rho(M)}{\log |q|},
$$

where $\rho(M)$ denotes the spectral radius of certain contact matrix $M$, and the corresponding measure is positive and finite.

When $A$ is a similarity, we can improve the well-known Hausdorff dimension formula of the boundary in the following simpler way.

Theorem 1.4 Let $A \in M_{2}(\mathbb{Z})$ be an expanding similarity with characteristic polynomial $f(x)=x^{2}+p x+q$ and $T=T(A, \mathcal{D})$ be a disk-like CC tile. Then

$$
\operatorname{dim}_{H}(\partial T)=\frac{2 \log \rho}{\log |q|}
$$

where $\rho$ is the largest positive zero of the cubic polynomial $x^{3}-(|p|-1) x^{2}-$ $(|q|-|p|) x-|q|$. 
The rest of the paper is organized as follows: In Sect. 2 , we identify $\partial T$ with a sofic system by constructing a neighbor graph and prove Theorem 1.2. In Sect. 3, we consider $\partial T$ as a graph-directed set and prove Theorems 1.3 and 1.4. Finally all neighbor graphs, graph-directed sets and contact matrices corresponding to different characteristic polynomials $f(x)$ are listed in Appendices 1-3 for easy reference.

\section{Sofic System and Number System}

We first introduce some terminology of symbolic dynamics from [27]. Let $\mathcal{G}=$ $\mathcal{G}(\mathcal{V}, \mathcal{E})$ be a directed graph where $\mathcal{V}$ is the set of vertices and $\mathcal{E}$ the set of edges. Let $\mathcal{A}$ be a finite set (called alphabet). If there exists a mapping (called labeling) $\mathcal{L}: \mathcal{E} \rightarrow \mathcal{A}$, then the ordered pair $\mathbf{G}=(\mathcal{G}, \mathcal{L})$ is called a labeled directed graph. All the infinite paths $\xi=e_{1} e_{2} e_{3} \ldots$ on $\mathcal{G}$ constitute the so-called edge shift $\mathbf{X}_{\mathcal{G}}$. Define the label of the path $\xi$ by

$$
\mathcal{L}_{\infty}(\xi):=\mathcal{L}\left(e_{1}\right) \mathcal{L}\left(e_{2}\right) \mathcal{L}\left(e_{3}\right) \ldots \in \mathcal{A}^{\mathbb{N}}
$$

Here $\mathcal{A}^{\mathbb{N}}$ is called the full shift of $\mathcal{A}$. The set of all such labels is denoted by

$$
\mathbf{X}_{\mathbf{G}}=\left\{x \in \mathcal{A}^{\mathbb{N}}: x=\mathcal{L}_{\infty}(\xi) \text { for some } \xi \in \mathbf{X}_{\mathcal{G}}\right\}
$$

Any subset of $\mathcal{A}^{\mathbb{N}}$ which can be defined by a labeled directed graph as above, is called a sofic shift or sofic system [11,27]. Weiss [33] coined the term sofic which is derived from the Hebrew word for finite [27].

Let $D=\{0,1, \ldots,|q|-1\}$ and the difference set $\Delta D:=D-D$, then the CC digit set $\mathcal{D}=D v$ and $\Delta \mathcal{D}:=\mathcal{D}-\mathcal{D}=\Delta D v$. Without loss of generality, we assume the digit set $\mathcal{D}$ is primitive, i.e., the lattice $\mathcal{J}$ generated by $\mathcal{D}$ and $A \mathcal{D}$ in $\mathbb{Z}^{2}$ is equal to $\mathbb{Z}^{2}$. For otherwise, there exists an invertible $B \in M_{2}(\mathbb{Z})$ such that $\tilde{\mathcal{D}}=B^{-1} \mathcal{D} \subset \mathbb{Z}^{2}$ is primitive and $T(A, \mathcal{D})=B T(\tilde{A}, \tilde{\mathcal{D}})$ where $\tilde{A}=B^{-1} A B \in M_{2}(\mathbb{Z})[21]$ and we can consider $\tilde{A}, \tilde{\mathcal{D}}$ instead. Hence we set $\mathbb{Z}^{2}=\{\gamma v+\delta A v: \gamma, \delta \in \mathbb{Z}\}$. It is easy to see that $T+\ell$ where $\ell \in \mathbb{Z}^{2}$ is a neighbor of $T$ if and only if $\ell \in T-T$. More precisely, $\ell$ can be expressed as

$$
\ell=\sum_{i=1}^{\infty} b_{i} A^{-i} v \in T-T, \quad b_{i} \in \Delta D
$$

The following is a neighbor-generating formula which plays a key role in constructing the labeled directed graph for the boundary.

Lemma 2.1 ([24]) Suppose $T+\ell$ is a neighbor of $T$ with $\ell=\gamma v+\delta A v=$ $\sum_{i=1}^{\infty} b_{i} A^{-i} v$, then we get another neighbor $T+\ell^{\prime}$ satisfying $\ell^{\prime}=A \ell-b_{1} v=$ $\gamma^{\prime} v+\delta^{\prime} A v$ with $\gamma^{\prime}=-\left(q \delta+b_{1}\right)$ and $\delta^{\prime}=\gamma-p \delta$.

Inductively, we can construct a sequence of neighbors: $\left\{T+\ell_{n}\right\}_{n=0}^{\infty}$ where $\ell_{0}=\ell$ and $\ell_{n+1}=A \ell_{n}-b_{n+1} v$. 
Let $T$ be a disk-like CC tile and $T_{\ell}=T \cap(T+\ell)$ for any $\ell \in \mathbb{Z}^{2}$. Let $\mathcal{V}=\left\{\ell \in \mathbb{Z}^{2}\right.$ : $\ell \neq 0$ and $\left.T \cap T_{\ell} \neq \emptyset\right\}$. Then the boundary of $T$ can be written as

$$
\partial T=\bigcup_{\ell \in \mathcal{V}} T_{\ell}
$$

Define an edge set $\mathcal{E}:=\left\{e=\left(\ell, \ell^{\prime}\right): \ell, \ell^{\prime} \in \mathcal{V}\right.$ and $\ell^{\prime}=A \ell-b_{1} v$ for some $\left.b_{1} \in \Delta D\right\}$ and a labeling $\mathcal{L}: \mathcal{E} \rightarrow \mathcal{A}$ by $\mathcal{L}(e)=b_{1}$ where $\mathcal{A}=\Delta D$. Then by the definition above, $\mathbf{G}=(\mathcal{G}, \mathcal{L})$ is a labeled directed graph and it determines a sofic shift. We call $\mathbf{G}$ the neighbor graph of $T$.

Proposition 2.2 Let $\mathbf{G}$ be the neighbor graph of a CC disk-like tile T. If $x=$ $\sum_{i=1}^{\infty} a_{i} A^{-i} v=\ell+\sum_{i=1}^{\infty} a_{i}^{\prime} A^{-i} v \in T_{\ell}$ where $a_{i}, a_{i}^{\prime} \in D$, then $\left\{b_{i}:=a_{i}-a_{i}^{\prime}\right\}_{i=1}^{\infty}$ is the sequence of labeling of the edges of an infinite path starting at $\ell$ (or simply called $a$ label sequence starting at $\ell$ ). Conversely, any label sequence $\left\{b_{i}\right\}_{i=1}^{\infty}$ (with $\left.b_{i} \in \Delta D\right)$ starting at $\ell$ defines a set

$$
\left\{x: x=\sum_{i=1}^{\infty} a_{i} A^{-i} v=\ell+\sum_{i=1}^{\infty} a_{i}^{\prime} A^{-i} v, a_{i}-a_{i}^{\prime}=b_{i}, a_{i}, a_{i}^{\prime} \in D \text { for } i=1,2, \ldots\right\}
$$

of boundary points of $T$.

Proof Since $\ell=\sum_{i=1}^{\infty} b_{i} A^{-i} v$ with $b_{i}=a_{i}-a_{i}^{\prime}$, by Lemma 2.1, we have a sequence of neighbors $\left\{T+\ell_{n}\right\}_{n=0}^{\infty}$ where $\ell_{0}=\ell$ and $\ell_{n+1}=A \ell_{n}-b_{n+1} v$, hence $\left\{b_{i}\right\}_{i=1}^{\infty}$ is a label sequence starting at $\ell$ by the definition.

Conversely, if $\ell=\sum_{i=1}^{\infty} b_{i} A^{-i} v$ where $b_{i} \in \Delta D$, then $b_{i}=a_{i}-a_{i}^{\prime}$ for $a_{i}, a_{i}^{\prime} \in D$ and $\ell=\sum_{i=1}^{\infty}\left(a_{i}-a_{i}^{\prime}\right) A^{-i} v$. It follows that

$$
x=\sum_{i=1}^{\infty} a_{i} A^{-i} v=\ell+\sum_{i=1}^{\infty} a_{i}^{\prime} A^{-i} v \in T \cap(T+\ell)=T_{\ell}
$$

We can verify whether the origin 0 is a boundary point of $T$ in the following way.

Corollary 2.3 $0 \in \partial T$ if and only if there exists an infinite path in $\mathbf{G}$ with all edge labels either non-positive or non-negative.

Proof Suppose $0 \in T \cap(T+\ell)$ for some neighbor $T+\ell$. Putting $a_{i}=0$ for all $i$ into (2.2), we have

$$
\ell=\sum_{i=1}^{\infty}\left(-a_{i}^{\prime}\right) A^{-i} v
$$

Since $a_{i}^{\prime} \in D$, the label sequence $\left\{b_{i}=-a_{i}^{\prime}\right\}_{i=1}^{\infty}$ starting at $\ell$ has all labels non-positive. Similarly $\left\{b_{i}^{\prime}=a_{i}\right\}_{i=1}^{\infty}$ is a sequence starting at $-\ell$ with all labels non-negative. By reversing the argument, we can prove the converse. 
Table 1 Relation among all neighbors of $T$ associated with $f(x)=x^{2}+p x+q, p, q \geq 2$, $2 p \leq q+2$ (excluding $p=q=2$ )

\begin{tabular}{lll}
\hline$\ell$ & $b_{1}$ & $\ell^{\prime}$ \\
\hline$v$ & $-(p-1)$ & $A v+(p-1) v$ \\
& $-p$ & $A v+p v$ \\
$A v+(p-1) v$ & $-(q-p)$ & $-A v-p v$ \\
& $-(q-p+1)$ & $-A v-(p-1) v$ \\
$A v+p v$ & $-(q-1)$ & $-v$ \\
$-v$ & $p-1$ & $-A v-(p+1) v$ \\
& $p$ & $-A v-p v$ \\
$-A v-(p-1) v$ & $q-p$ & $A v+p v$ \\
& $q-p+1$ & $A v+(p-1) v$ \\
$-A v-p v$ & $q-1$ & $v$ \\
\hline
\end{tabular}

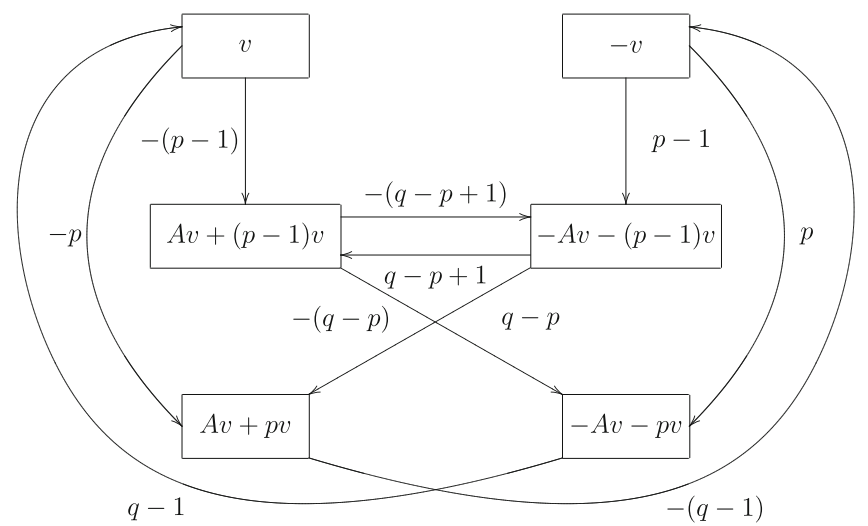

Fig. 1 The neighbor graph of $T$ associated with $f(x)=x^{2}+p x+q, p, q \geq 2,2 p \leq q+2$ (excluding $q=p=2)$

In fact, we can determine the neighbor graph $\mathbf{G}$ for any disk-like CC tile $T$. Let us take the case of $f(x)=x^{2}+p x+q, p, q \geq 2$ (excluding $p=q=2$ ) as an example. By Theorem 1.1, $T$ is a hexagonal tile with six neighbors [24] and

$$
\mathcal{V}=\{ \pm v, \pm(A v+(p-1) v), \pm(A v+p v)\}
$$

In view of the definition of $\mathcal{E}$, if $\ell=v$ we take $b_{1}=-p$ and $\ell^{\prime}=A v+p v$ or $b_{1}=-(p-1)$ and $\ell^{\prime}=A v+(p-1) v$; if $\ell=A v+p v$, using $f(A) v=0$, we have $b_{1}=-(q-1)$ and $\ell^{\prime}=-v$. Proceeding similarly with all $\ell$, we obtain Table 1 . Then we establish the neighbor graph (Fig. 1). The neighbor graphs corresponding to other $f(x)$ are given in Appendix 1.

Following [20], we let $\mathcal{D}_{A, k}=\left\{\sum_{i=0}^{k-1} a_{i} A^{i} v: a_{i} \in D\right\}, \Delta \mathcal{D}_{A, k}=\mathcal{D}_{A, k}-\mathcal{D}_{A, k}=$ $\left\{\sum_{i=0}^{k-1} b_{i} A^{i} v: b_{i} \in \Delta D\right\}$ and $\mathcal{D}_{A, \infty}=\bigcup_{k=1}^{\infty} \mathcal{D}_{A, k}$. 
Proposition 2.4 Let $T$ be a disk-like $C C$ tile and $T+\ell$ a neighbor. Then $\ell=$ $\sum_{i=0}^{k} b_{i} A^{i} v \in \Delta \mathcal{D}_{A, k+1}$ for some $k \in \mathbb{Z}$ with $b_{k} \in\{-1,1\}, b_{i} \in \Delta D$ for $0 \leq i<k$. When $f(x)=x^{2} \pm 2 x+2, k=3$; and $k=1$ otherwise.

Proof It follows from (2.3) that $\ell \in \Delta \mathcal{D}_{A, 2}$ excluding the case of $f(x)=x^{2} \pm 2 x+2$. For $f(x)=x^{2} \pm 2 x+2$, we have $A v \pm 2 v=A^{3} v \pm A^{2} v+A v \in \Delta \mathcal{D}_{A, 4}$ by using $(A \mp I) f(A)=0$.

A more desirable property is that any $\ell \in \mathbb{Z}^{2}$ can be expressed as $\ell=\sum_{i=0}^{k} a_{i} A^{i} v \in$ $\mathcal{D}_{A, k+1}$ (instead of $\Delta \mathcal{D}_{A, k+1}$ ) for some $k \in \mathbb{Z}$ with $a_{k}=1$ and $a_{i} \in D$ where $0 \leq i<k$. But this is not always the case. This property is closely related to a number system defined below (see also [29]).

Definition 2.5 Let $A \in M_{2}(\mathbb{Z})$ be expanding and $\mathcal{D}$ be a $C C$ digit set. The selfaffine pair $(A, \mathcal{D})$ is said to be a number system if for any $\ell \in \mathbb{Z}^{2}$, it has a unique representation $\ell=\sum_{i=0}^{k} A^{i} v_{i}^{\prime}$ with $v_{i}^{\prime} \in \mathcal{D}$.

For convenience, we sometimes write a point of the form $x=\sum_{i=1}^{\infty} a_{i} A^{-i} v \in \mathbb{R}^{2}$ as radix expansion: $0 . a_{1} a_{2} a_{3} \ldots$ An overbar denotes repeating digits as in $0.12 \overline{301}=$ $0.12301301301 \ldots$ Likewise, $a_{-2} a_{-1} a_{0} \cdot a_{1} a_{2} a_{3} \ldots$ represents a point $a_{-2} A^{2} v+$ $a_{-1} A v+a_{0} v+\sum_{i=1}^{\infty} a_{i} A^{-i} v$. Note that shifting a radix place to the left means multiplying $A$ to $x$. When $x$ is on the boundary of $T$, the radix expansion of $x$ is not unique. Now we give some equivalent conditions for the self-affine pair $(A, \mathcal{D})$ to be a number system.

Theorem 2.6 Let $T=T(A, \mathcal{D})$ be a disk-like CC tile. Then the following are equivalent:

(i) $(A, \mathcal{D})$ is a number system.

(ii) $0 \in T^{\circ}$.

(iii) $f(x)=x^{2}+p x+q$ with $-1 \leq p$ and $q \geq 2$.

(iv) For all neighbors $T+\ell, \ell=\sum_{i=0}^{k} a_{i} A^{i} v \in \mathcal{D}_{A, k+1}$ for some $k \in \mathbb{Z}$ with $a_{k}=1$ and $a_{i} \in D$ where $0 \leq i<k$.

Proof (i) $\Rightarrow$ (ii) Suppose $0 \notin T^{\circ}$. Then $0 \in T \cap(T+\ell)$ for some $\ell \in \mathbb{Z}^{2} \backslash\{0\}$. Since $(A, \mathcal{D})$ is a number system, $\ell=\sum_{i=-k}^{0} a_{i} A^{-i} v$ with $a_{i} \in D$ and $a_{-k}>0$. Hence $0=a_{-k} a_{-(k-1)} \ldots a_{-1} a_{0} \cdot a_{1} a_{2} a_{3} \ldots$ Shifting the radix point $k$ places to the left, we get $0=a_{-k} \cdot a_{-(k-1)} \ldots a_{-1} a_{0} a_{1} a_{2} a_{3} \ldots$ That means $T+a_{-k} v$ is a neighbor of $T$. By Proposition 2.4, $a_{-k}=1$. Hence 0 corresponds to an infinite path starting at $v$ with non-positive labels $b_{i}=-a_{i}$. But by checking all the neighbor graphs in Appendix 1, we find no such path.

(ii) $\Rightarrow$ (i) It suffices to show that $\mathbb{Z}^{2} \subset \mathcal{D}_{A, \infty}$. By the lattice tiling property, 0 is the only lattice point in $T$, i.e., $\mathbb{Z}^{2} \cap T=\{0\}$. It follows that $\mathbb{Z}^{2} \cap A^{n} T=\sum_{i=0}^{n-1} A^{i} \mathcal{D}=$ $\mathcal{D}_{A, n}$ for $n \geq 1$. If $\ell \in \mathbb{Z}^{2}$, there exists a large integer $n$ such that $\ell \in A^{n} T$ as $0 \in T^{\circ}$, then $\ell \in \mathcal{D}_{A, n} \subset \mathcal{D}_{A, \infty}$.

(ii) $\Leftrightarrow$ (iii) By inspecting all neighbor graphs in Appendix 1, we find that in each graph corresponding to $f(x)=x^{2}+p x+q$ with $-1 \leq p$ and $q \geq 2$, there exists no infinite path with edge labels either all non-positive or all non-negative, hence $0 \in T^{\circ}$ 
Table 2 Infinite paths representing a boundary point 0

\begin{tabular}{lll}
\hline$f(x)$ & Neighbor & Path \\
\hline$x^{2}-2 x+2$ & $A v-v$ & $\overline{(-1)}$ \\
$x^{2}-p x-q$ & $-A v+v$ & $\overline{1}$ \\
$x^{2}+p x-q$ & $-v$ & $\overline{p(q-1)}$ \\
& $A v+(p+1) v$ & $\overline{(-p)[-(q-1)]}$ \\
$x^{2}-q$ & $-A v-(p+1) v$ & $\overline{[q-p-1)}$ \\
& $v$ & $\frac{\overline{(q-1)}-1)]}{0[-(q-1)]}$ \\
& $-v$ & $\frac{\overline{(q-1)}}{[-(q-1)]}$ \\
$x^{2}-p x+q$ & $A v+v$ & $\frac{\overline{[-(q-p+1)]}}{(q-p+1)}$ \\
\hline
\end{tabular}

by Corollary 2.3. In every other case, there always exists such a path. All these paths are listed in Table 2.

(iii) $\Rightarrow$ (iv) Let $f(x)$ be one of the cases: $x^{2}+q, x^{2}+x+q, x^{2}+p x+q(p \geq 2$, excluding $p=q=2$ ), $x^{2}+2 x+2, x^{2}-x+q$, where $p \geq 0$ and $q \geq 2$. In each case, we can rewrite their neighbors as the desired form in (iv). By using $0=f(A) v$, $0=(A-I) f(A) v, 0=(A+I) f(A) v$, we have

Case (1) $f(x)=x^{2}+q . \quad A v-v=A^{2} v+A v+(q-1) v,-v=A^{2} v+(q-1) v$, $-A v=A^{3} v+(q-1) A v,-A v+v=A^{3} v+(q-1) A v+v,-A v-v=$ $A^{3} v+A^{2} v+(q-1) A v+(q-1) v$.

Case (2) $f(x)=x^{2}+x+q . \quad-v=A^{2} v+A v+(q-1) v,-A v=A^{3} v+A^{2} v+$ $(q-1) A v,-A v-v=A^{2} v+(q-1) v$.

Case (3) $f(x)=x^{2}+p x+q(p \geq 2) .-v=A^{2} v+p A v+(q-1) v,-A v-(p-1)$ $v=A^{2} v+(p-1) A v+(q-p+1) v,-A v-p v=A^{2} v+(p-1) A v+(q-p) v$.

Case (4) $f(x)=x^{2}+2 x+2 . \quad A v+2 v=A^{3} v+A^{2} v+A v,-v=A^{4} v+A^{3} v+$ $A^{2} v+v,-A v-v=A^{2} v+A v+v,-A v-2 v=A^{2} v+A v$.

Case (5) $f(x)=x^{2}-x+q . A v-v=A^{2} v+(q-1) v,-v=A^{3} v+$ $(q-1) A v+(q-1) v,-A v=A^{4} v+(q-1) A^{2} v+(q-1) A v,-A v+v=$ $A^{4} v+(q-1) A^{2} v+(q-1) A v+v$.

(iv) $\Rightarrow$ (ii) Suppose $0 \notin T^{\circ}$. By the same argument as in the proof of (i) $\Rightarrow$ (ii) above, there should be an infinite path in the neighbor graph starting at $v$ with edge labels all non-positive. But we find no such path by inspecting all the neighbor graphs in Appendix 1.

Remark 2.7 Gilbert [13] obtained some related results in the context of quadratic number fields. We conjecture that Theorem 2.6 can be extended to non-disk-like tiles. 


\section{Dimension of the Boundary of $T$}

For a directed graph $\mathcal{G}=\mathcal{G}(\mathcal{V}, \mathcal{E})$ where $\mathcal{V}=\left\{v_{1}, v_{2}, \ldots, v_{m}\right\}$, we write $\mathcal{E}_{i, j}$ for the set of edges from vertex $v_{i}$ to vertex $v_{j}$, and we add a contraction mapping $F_{e}: \mathbb{R}^{2} \rightarrow \mathbb{R}^{2}$ for each edge $e \in \mathcal{E}$. Then the family of contractions $\left\{F_{e}: e \in \mathcal{E}\right\}$ is called a graphdirected iterated function system (GIFS) and there exists a unique family of non-empty compact subsets $E_{1}, \ldots, E_{m}$ of $\mathbb{R}^{2}[9,28]$ such that

$$
E_{i}=\bigcup_{j=1}^{m} \bigcup_{e \in \mathcal{E}_{i, j}} F_{e}\left(E_{j}\right)
$$

We call $E:=\bigcup_{i=1}^{m} E_{i}$ a graph-directed set. Define $M=\left(M_{i j}\right)_{1 \leq i, j \leq m}$ as the contact matrix [15] of $\mathcal{G}$ with $M_{i j}=\# \mathcal{E}_{i, j}$ counting the number of edges from $v_{i}$ to $v_{j}$.

The GIFS $\left\{F_{e}: e \in \mathcal{E}\right\}$ is said to satisfy the open set condition (OSC) if there exists a family of open sets $\left\{O_{1}, \ldots, O_{m}\right\}$ such that

$$
O_{i} \supset \bigcup_{j=1}^{m} \bigcup_{e \in \mathcal{E}_{i, j}} F_{e}\left(O_{j}\right) \quad \text { for } i=1,2, \ldots, m
$$

with disjoint unions, i.e., $F_{e}\left(O_{j}\right) \cap F_{e^{\prime}}\left(O_{j^{\prime}}\right)=\emptyset$ whenever $(e, j) \neq\left(e^{\prime}, j^{\prime}\right)$. With this OSC, we then can compute the dimension of the graph-directed set.

In this section, we first identify the boundary of $T$ with a graph-directed set by making use of the well-known method [17,31], then calculate its dimension in the self-affine case and the self-similar case, respectively.

Proposition 3.1 Let $\ell=\gamma v+\delta A v, \ell^{\prime}=\gamma^{\prime} v+\delta^{\prime} A v \in \mathcal{V}$ such that $\ell^{\prime}=A \ell-b_{1} v$ for some $b_{1} \in \Delta D$, then

$$
A^{-1}\left(T_{\ell^{\prime}}+j v\right) \subset T_{\ell} \text { for all } j \in I_{b_{1}}:=\left\{\begin{array}{l}
\left\{b_{1}, b_{1}+1, \ldots, q-1\right\} \text { if } b_{1} \geq 0 \\
\left\{0,1, \ldots, q-1+b_{1}\right\} \text { if } b_{1}<0 .
\end{array}\right.
$$

Moreover,

$$
T_{\ell}=\bigcup_{\ell^{\prime} \in B_{\ell}} \bigcup_{j \in I_{b_{1}}} A^{-1}\left(T_{\ell^{\prime}}+j v\right)
$$

where $B_{\ell}:=\left\{\ell^{\prime \prime} \in \mathcal{V}: \ell^{\prime \prime}=A \ell-b_{1}^{\prime} v\right.$ for some $\left.b_{1}^{\prime} \in \Delta D\right\}$. Hence the boundary $\partial T=\bigcup_{\ell \in \mathcal{V}} T_{\ell}$ is a graph-directed set.

Proof When $b_{1} \geq 0$, if $x \in T_{\ell^{\prime}}$ then the radix expansion is

$$
x=0 . c_{1} c_{2} c_{3} \ldots=\delta^{\prime} \gamma^{\prime} \cdot c_{1}^{\prime} c_{2}^{\prime} c_{3}^{\prime} \ldots
$$

It follows from Lemma 2.1 and $0=A^{-1} f(A) v$ that

$$
A^{-1} x+\left(b_{1}+k\right) A^{-1} v=0 .\left(b_{1}+k\right) c_{1} c_{2} c_{3} \ldots=\delta \gamma \cdot k c_{1}^{\prime} c_{2}^{\prime} c_{3}^{\prime} \ldots \in T_{\ell}
$$

for $k=0,1, \ldots, q-1-b_{1}$. The case when $b_{1}<0$ can be proved similarly. 
For the second part, we only need to show

$$
T_{\ell} \subset \bigcup_{\ell^{\prime} \in B_{\ell}} \bigcup_{j \in I_{b_{1}}} A^{-1}\left(T_{\ell^{\prime}}+j v\right) .
$$

Let $y=0 . a_{1} a_{2} a_{3} \ldots=\delta \gamma \cdot a_{1}^{\prime} a_{2}^{\prime} a_{3}^{\prime} \ldots \in T_{\ell}$. It follows that $A y-a_{1} v=$ $0 . a_{2} a_{3} a_{4} \ldots=\delta \gamma\left(a_{1}^{\prime}-a_{1}\right) \cdot a_{2}^{\prime} a_{3}^{\prime} \ldots \in T_{\ell^{\prime}}$, where $\ell^{\prime}=A \ell-\left(a_{1}-a_{1}^{\prime}\right) v$. This implies $y \in A^{-1}\left(T_{\ell^{\prime}}+a_{1} v\right)$. By definition, we see that $a_{1} \in I_{b_{1}}$ for $b_{1}=a_{1}-a_{1}^{\prime}$.

It should be mentioned that the graph for the GIFS comes from the neighbor graph by adding more edges, or equivalently the neighbor graph is a reduced graph for the GIFS. The following example about Fig. 1 can illustrate their relationship. All the other cases are given in Appendix 2.

Example 3.2 Consider the case $f(x)=x^{2}+p x+q(p, q \geq 2$, excluding $p=q=2)$. When $\ell=v$, from Table 1 we have $B_{\ell}=B_{v}=\{A v+p v, A v+(p-1) v\}$. When $\ell^{\prime}=$ $A v+p v, b_{1}=-p$ and $I_{-p}=\{0,1,2, \ldots, q-1-p\}$; when $\ell^{\prime}=A v+(p-1) v, b_{1}=$ $-(p-1)$ and $I_{-(p-1)}=\{0,1,2, \ldots, q-p\}$. Thus by Proposition 3.1, the first set equation comes out. Similarly the other five can be deduced. For simplicity we let $u_{1}=v, u_{2}=A v+(p-1) v, u_{3}=A v+p v$. Then the sets $T_{ \pm u_{1}}, T_{ \pm u_{2}}, T_{ \pm u_{3}}$, representing $\partial T$ satisfy

$$
\begin{aligned}
A T_{u_{1}} & =\bigcup_{j=0}^{q-p}\left(T_{u_{2}}+j v\right) \cup \bigcup_{j=0}^{q-p-1}\left(T_{u_{3}}+j v\right), \\
A T_{u_{2}} & =\bigcup_{j=0}^{p-2}\left(T_{-u_{2}}+j v\right) \cup \bigcup_{j=0}^{p-1}\left(T_{-u_{3}}+j v\right), \\
A T_{u_{3}} & =T_{-u_{1}}, \\
A T_{-u_{1}} & =\bigcup_{j=p-1}^{q-1}\left(T_{-u_{2}}+j v\right) \cup \bigcup_{j=p}^{q-1}\left(T_{-u_{3}}+j v\right), \\
A T_{-u_{2}} & =\bigcup_{j=q-p+1}^{q-1}\left(T_{u_{2}}+j v\right) \cup \bigcup_{j=q-p}^{q-1}\left(T_{u_{3}}+j v\right), \\
A T_{-u_{3}} & =T_{u_{1}}+(q-1) v .
\end{aligned}
$$

The Hausdorff dimension $\left(\operatorname{dim}_{H}\right)$ (see e.g., $\left.[9,10]\right)$ is the most common and important dimension in fractal geometry. The case of self-similar sets has been studied extensively with or without separation conditions. However the case of self-affine sets is still hard to handle. Recently, He and Lau [16] defined the generalized Hausdorff dimension $\left(\operatorname{dim}_{H}^{\omega}\right)$ and Hausdorff measure $\left(\mathcal{H}_{\omega}^{s}\right)$ for self-affine fractals by replacing the Euclidean norm with a pseudo-norm $\omega$ for which the expanding matrix $A$ becomes a similarity:

$$
\omega(A x)=|\operatorname{det} A|^{1 / 2} \omega(x) .
$$


Under this setting, most of the basic properties for the self-similar sets can be carried to the self-affine sets. Moreover, Luo and Yang [26] extended this technique to the self-affine GIFS and obtained a dimension formula of the graph-directed set we need.

Proposition 3.3 ([26]) For the GIFS as in (3.1) with the affine mappings $F_{e}(x)=$ $A^{-1}\left(x+d_{e}\right)$ where $A$ is an expanding matrix and $|\operatorname{det} A|=|q|$, let $\rho(M)$ be the spectral radius of the contact matrix $M$. If the OSC holds, then $s=\operatorname{dim}_{H}^{\omega} E=$ $2 \log \rho(M) / \log |q|$ and $0<\mathcal{H}_{\omega}^{s}(E)<\infty$.

By using this, we can establish our first-dimensional result about the boundary of $T$ as follows.

Theorem 3.4 The generalized Hausdorff dimension of the boundary of disk-like CC tile $T$ is

$$
\operatorname{dim}_{H}^{\omega}(\partial T)=2 \log \rho(M) / \log |q|
$$

and the corresponding measure is positive and finite.

Proof From Propositions 3.1 and 3.3, it suffices to show the GIFS representing the boundary of $T$ satisfies the OSC. Replacing $T_{\ell}$ by $(T+\ell)^{\circ}$, we can check the OSC holds case by case. We illustrate the idea by proving the case $f(x)=x^{2}+p x+q(p \geq$ 2, $q \geq 2$, excluding $p=q=2$ ). In view of Example 3.2, we need to show

$$
\begin{aligned}
A\left(T+u_{1}\right)^{\circ} \supset \bigcup_{j=0}^{q-p}\left(\left(T+u_{2}\right)^{\circ}+j v\right) \cup \bigcup_{j=0}^{q-p-1}\left(\left(T+u_{3}\right)^{\circ}+j v\right), \\
A\left(T+u_{2}\right)^{\circ} \supset \bigcup_{j=0}^{p-2}\left(\left(T-u_{2}\right)^{\circ}+j v\right) \cup \bigcup_{j=0}^{p-1}\left(\left(T-u_{3}\right)^{\circ}+j v\right), \\
A\left(T+u_{3}\right)^{\circ} \supset\left(T-u_{1}\right)^{\circ}, \\
A\left(T-u_{1}\right)^{\circ} \supset \bigcup_{j=p-1}^{q-1}\left(\left(T-u_{2}\right)^{\circ}+j v\right) \cup \bigcup_{j=p}^{q-1}\left(\left(T-u_{3}\right)^{\circ}+j v\right), \\
A\left(T-u_{2}\right)^{\circ} \supset \bigcup_{j=q-p+1}^{q-1}\left(\left(T+u_{2}\right)^{\circ}+j v\right) \cup \bigcup_{j=q-p}^{q-1}\left(\left(T+u_{3}\right)^{\circ}+j v\right), \\
A\left(T-u_{3}\right)^{\circ} \supset\left(T+u_{1}\right)^{\circ}+(q-1) v, \quad
\end{aligned}
$$

with disjoint unions. Since $T$ is a $\mathrm{CC}$ tile, it follows that

$$
A T^{\circ} \supset \bigcup_{j=0}^{q-1}(T+j v)^{\circ}=\bigcup_{j=0}^{q-1}\left(T^{\circ}+j v\right)
$$

with disjoint union. By using (3.3) and $0=f(A) v=A^{2} v+p A v+q v$ extensively, we prove the first two set inequalities in the following. The remaining four can be verified similarly. 
For $j=0,1, \ldots, q-p$,

$$
\left(T+u_{2}\right)^{\circ}+j v=T^{\circ}+(p-1+j) v+A v \subset A\left(T+u_{1}\right)^{\circ} .
$$

For $j=0,1, \ldots, q-p-1$,

$$
\left(T+u_{3}\right)^{\circ}+j v=T^{\circ}+(p+j) v+A v \subset A\left(T+u_{1}\right)^{\circ} .
$$

For $j=0,1, \ldots, p-2$,

$$
\begin{aligned}
\left(T-u_{2}\right)^{\circ}+j v & =T^{\circ}+(j-p+1) v-A v \\
& =T^{\circ}+(q+j-p+1) v+A^{2} v+(p-1) A v \\
& \subset A\left(T+u_{2}\right)^{\circ} .
\end{aligned}
$$

For $j=0,1, \ldots, p-1$,

$$
\begin{aligned}
\left(T-u_{3}\right)^{\circ}+j v & =T^{\circ}+(j-p) v-A v \\
& =T^{\circ}+(q+j-p) v+A^{2} v+(p-1) A v \\
& \subset A\left(T+u_{2}\right)^{\circ} .
\end{aligned}
$$

By the same way, all the other cases follow and hence the theorem is proved.

In the rest of this section, we will find the exact value of Hausdorff dimension $\operatorname{dim}_{H}(\partial T)$ for certain particular cases that $A$ is a similarity. We state the simplest one first.

Proposition 3.5 Let $A \in M_{2}(\mathbb{Z})$ be expanding with characteristic polynomial $f(x)=x^{2}+q(|q| \geq 2)$ and $T(A, \mathcal{D})$ a disk-like CC tile. Then $\operatorname{dim}_{H}(\partial T)=1$.

Proof By Theorem 1.1, $T$ is a square tile (parallelogram). Hence $\operatorname{dim}_{H}(\partial T)=1$.

Geometrically, a similarity is a multiple of either a reflection or a rotation. We call the former a scaled reflection and the latter a scaled rotation; algebraically, a similarity is a multiple of an orthogonal matrix. The case that $A$ is a scaled reflection is solved already as its characteristic polynomial is of the form $f(x)=x^{2}-q(q>0)$. So we focus our attention on those $A$ that are scaled rotations.

Lemma 3.6 Let A be a scaled rotation. Then its characteristic polynomial has positive constant term and A has either two distinct non-real eigenvalues or two equal real eigenvalues.

Proof Let $A=\left(\begin{array}{cc}r \cos \theta & -r \sin \theta \\ r \sin \theta & r \cos \theta\end{array}\right)$. The characteristic polynomial is given by $x^{2}-2 r \cos \theta x+r^{2}$. It has two equal real zeros when $\theta=0$ or $\pi$ and two distinct non-real zeros otherwise. 
Table 3 The contact matrix of $T$ associated with $f(x)=x^{2}+p x+q, p, q \geq 2,2 p \leq q+2$ (excluding $p=q=2$ )

\begin{tabular}{lllllll}
\hline & $v$ & $A v+(p-1) v$ & $A v+p v$ & $-v$ & $-A v-(p-1) v$ & $-A v-p v$ \\
\hline$v$ & 0 & $q-p+1$ & $q-p$ & 0 & 0 & 0 \\
$A v+(p-1) v$ & 0 & 0 & 0 & 0 & $p-1$ & $p$ \\
$A v+p v$ & 0 & 0 & 0 & 1 & 0 & 0 \\
$-v$ & 0 & 0 & 0 & 0 & $q-p+1$ & $q-p$ \\
$-A v-(p-1) v$ & 0 & $p-1$ & $p$ & 0 & 0 & 0 \\
$-A v-p v$ & 1 & 0 & 0 & 0 & 0 & 0 \\
\hline
\end{tabular}

The following dimension formula on the boundaries of self-similar tiles has been investigated in the literature by various methods (see $[8,31,32,17,23])$. We shall apply this formula to obtain our second dimensional result which is simpler than the known one.

Proposition 3.7 If $A$ is a similarity with $|\operatorname{det}(A)|=|q| \geq 2$, then the Hausdorff dimension of $\partial T$ is given by

$$
\operatorname{dim}_{H}(\partial T)=\log \rho(M) / \log r=2 \log \rho(M) / \log |q|,
$$

where $\rho(M)$ denotes the spectral radius of the contact matrix $M$ and $r=|q|^{1 / 2}$ is the expansion ratio of $A$.

Let $\ell, \ell^{\prime}, b_{1}$ and $B_{\ell}$ be defined as in Proposition 3.1. We first find the contact matrix $M$. Since $\mathcal{D}$ is a CC digit set, we have the entry $M_{\ell \ell^{\prime}}=\# I_{b_{1}}=q-\left|b_{1}\right|$ where $I_{b_{1}}$ is as in Proposition 3.1. Recall that $b_{1}$ is the label of the edge from $\ell$ to $\ell^{\prime}$. Hence we obtain the contact matrix $M$ of $T$ from its neighbor graph with different edge labels (i.e., replace $b_{1}$ by $q-\left|b_{1}\right|$ ).

Moreover, it is easy to see that there is a one-to-one correspondence between the contact matrix and the neighbor graph. For example, the contact matrix for the case $f(x)=x^{2}+p x+q(p, q \geq 2,2 p \leq q+2$ excluding $p=q=2)$ can be found in Table 3, and the related neighbor graph is shown by Fig. 1. The contact matrices for the other cases are given in Appendix 3.

If $M$ is irreducible (i.e., for each entry $M_{i j}$, there exists an integer $n \geq 0$ such that $\left(M^{n}\right)_{i j}>0$ ), then the spectral radius $\rho(M)=\lambda_{M}$ where $\lambda_{M}$ is the Perron-Frobenius eigenvalue of $M$ as stated in the following simplified version of the Perron-Frobenius Theorem.

Theorem 3.8 ([12,30]) Let $M$ be an irreducible non-negative matrix. Then there exists a positive eigenvalue $\lambda_{M}$ such that $\lambda_{M} \geq|\mu|$ for all eigenvalues $\mu$ of $M$. Moreover, $\lambda_{M}$ is a simple zero of the characteristic polynomial of $M$.

It is known that a contact matrix is irreducible if and only if the neighbor graph it represents is strongly connected. A directed graph is called strongly connected if for any two vertices $v_{i}, v_{j}$ there exists a path starting at $v_{i}$ and ending at $v_{j}$. 
Theorem 3.9 Let $A \in M_{2}(\mathbb{Z})$ be an expanding similarity with characteristic polynomial $f(x)=x^{2}+p x+q$ and $T(A, \mathcal{D})$ be a disk-like $C C$ tile. Then $\rho(M)$ is the largest positive zero of the cubic polynomial

$$
x^{3}-(|p|-1) x^{2}-(|q|-|p|) x-|q| .
$$

Hence $\operatorname{dim}_{H}(\partial T)=2 \log \rho(M) / \log |q|$.

Proof Since $|\operatorname{det}(A)|=|q|$, it is more convenient to work with $f(x)=x^{2} \pm p x \pm q$ $(p \geq 0, q \geq 2)$. Also we ignore those $f(x)$ of the form $f(x)=x^{2} \pm p x-q(p>0$, $q \geq 2$ ) as they cannot be characteristic polynomials of similarities (Lemma 3.6). We can see from Appendix 1 or 3 that the contact matrix is irreducible if and only if $f(x)=x^{2} \pm p x+q$ where $p>0$.

Case (1) $f(x)=x^{2}+p x+q$. The characteristic polynomial of the corresponding $M$ is $(x-1)\left(x^{2}+p x+q\right)\left[x^{3}-(p-1) x^{2}-(q-p) x-q\right]$. Notice that $\rho(M) \neq 1$. Indeed, if $\rho(M)=1$, then $\operatorname{dim}_{H}(\partial T)=0$, which implies $\partial T$ is totally disconnected (Proposition 2.5, [10]). This is not possible for the boundary of a topological disk. The zeros of $x^{2}+p x+q$ are either both negative or both non-real. Hence $\rho(M)$ is the largest positive real zero of $x^{3}-(p-1) x^{2}-(q-p) x-q$.

Case (2) $f(x)=x^{2}-p x+q$. The characteristic polynomial of the corresponding $M$ is $(x+1)\left(x^{2}-p x+q\right)\left[x^{3}-(p-1) x^{2}-(q-p) x-q\right]$. Since $f(x)$ cannot have unequal real zeros (Lemma 3.6), we have $p^{2}-4 q \leq 0$. When $p^{2}-4 q<0$, the zeros of $x^{2}-p x+q$ are non-real. Then $\rho(M)$ is the largest positive real zero of $x^{3}-(p-1) x^{2}-(q-p) x-q$. When $p^{2}-4 q=0$, the two zeros of $x^{2}-p x+q$ are equal. But the Perron-Frobenius eigenvalue should be a simple zero of the characteristic polynomial of $M$ (Theorem 3.8), so $\rho(M)$ is also the largest positive real zero of $x^{3}-(p-1) x^{2}-(q-p) x-q$.

Case (3) $f(x)=x^{2}+q$. The contact matrix $M$ is reducible. Its characteristic polynomial is $\left(x^{2}-q\right)\left(x^{2}+q\right)(x-1)(x+1)\left(x^{2}+1\right)$. We see that $\rho(M)=q^{1 / 2}$, which is the largest positive zero of $x^{3}+x^{2}-q x-q=\left(x^{2}-q\right)(x+1)$.

Case (4) $f(x)=x^{2}-q$. The contact matrix $M$ is also reducible and its characteristic polynomial is found to be $\left(x^{2}-q\right)^{2}(x+1)(x-1)^{3}$. As in the previous case, $\rho(M)=q^{1 / 2}$, which is also the largest positive zero of $x^{3}+x^{2}-q x-q$.

Remark 3.10 It is interesting to see that the signs of $p$ and $q$ do not matter in the calculation of $\operatorname{dim}_{H}(\partial T)$ when $A$ is a similarity. Notice also for the last two cases, $f(x)=x^{2}+q(|q| \geq 2)$, we have $\rho(M)=|q|^{1 / 2}$. It follows that $\operatorname{dim}_{H}(\partial T)=1$, as expected for the boundary of a parallelogram (Proposition 3.5).

We observe that $\operatorname{dim}_{H}(\partial T)$ is independent of the choice of the vector $v$ in the following sense.

Corollary 3.11 Let $A \in M_{2}(\mathbb{Z})$ be an expanding similarity with characteristic polynomial $f(x)=x^{2}+p x+q(|q| \geq 2)$. Let $\mathcal{D}=\mathcal{D}(v,|q|)$ and $\mathcal{D}^{\prime}=\mathcal{D}\left(v^{\prime},|q|\right)$ be two $C C$ digit sets such that each of $\{v, A v\}$ and $\left\{v^{\prime}, A v^{\prime}\right\}$ is an independent set. If $2|p| \leq|q+2|$, then

$$
\operatorname{dim}_{H}(\partial T(A, \mathcal{D}))=\operatorname{dim}_{H}\left(\partial T\left(A, \mathcal{D}^{\prime}\right)\right) .
$$


Proof As $2|p| \leq|q+2|$, both $T(A, \mathcal{D})$ and $T\left(A, \mathcal{D}^{\prime}\right)$ are disk-like CC tiles (Theorem 1.1). Hence the corollary follows from Theorem 3.9.

Remark 3.12 We conjecture that Theorem 3.9 and Corollary 3.11 are also valid when $2|p|>|q+2|$, i.e., $T$ is non-disk-like. The major difficulty in justifying these conjectures is that, in general, there is no upper bound on the number of neighbors of a non-disk-like CC tile [7].

Acknowledgments The authors would like to thank Professor Ka-Sing Lau for suggesting the question and reading an earlier version of the manuscript carefully. They are also grateful to the anonymous referees for their valuable comments and suggestions. The research is supported by STU Scientific Research Foundation for Talents (No. NTF12016).

\section{Appendix 1: Neighbor Graphs}

Let $f(x)=x^{2} \pm p x \pm q(p \geq 0, q \geq 2)$. The neighbor graphs of disk-like tiles are classified by $f(x)$ and listed in Figs. 2, 3, 4, 5, 6, 7, 8, 9, 10 and 11.

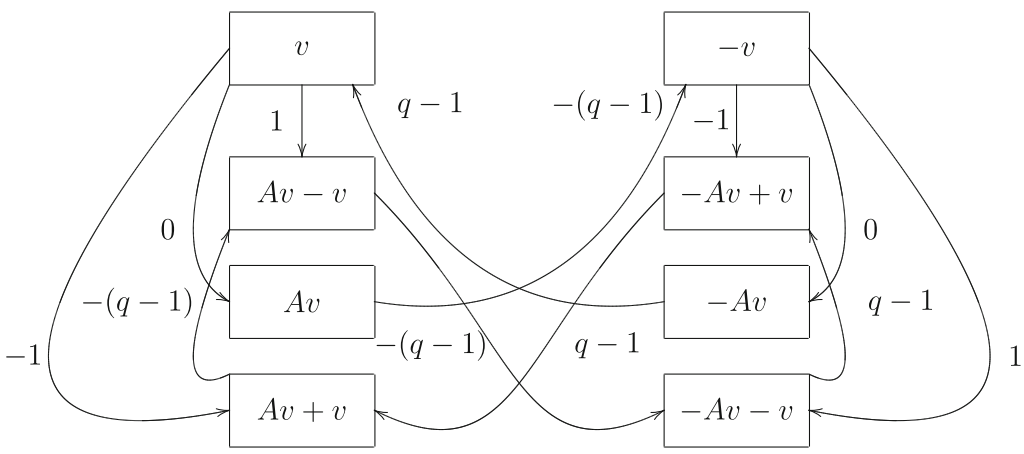

Fig. 2 The neighbor graph of $T$ associated with $f(x)=x^{2}+q$

Fig. 3 The neighbor graph of $T$ associated with $f(x)=x^{2}-q$

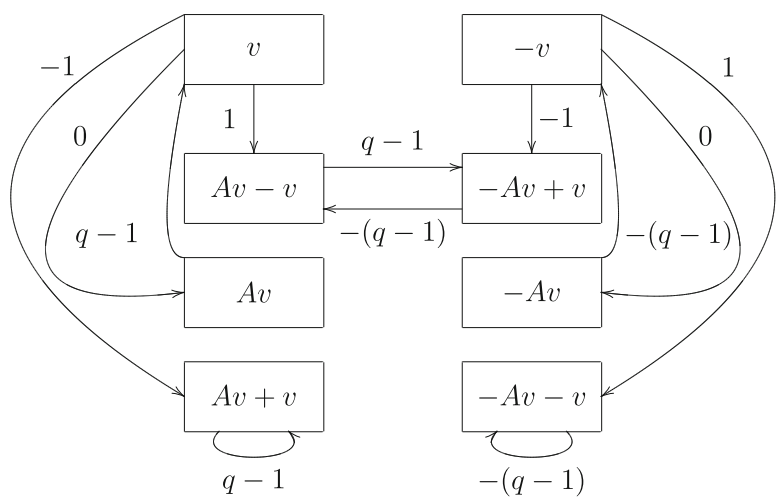




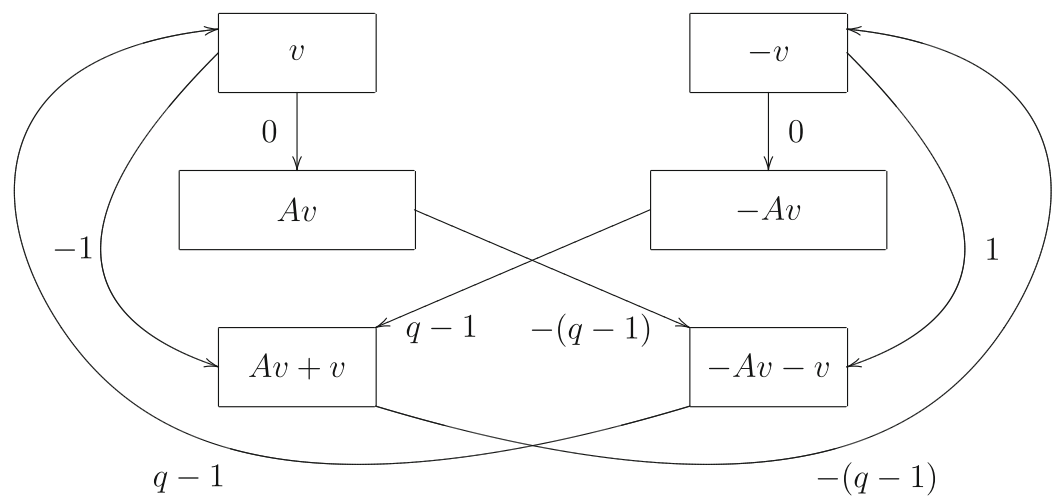

Fig. 4 The neighbor graph of $T$ associated with $f(x)=x^{2}+x+q$

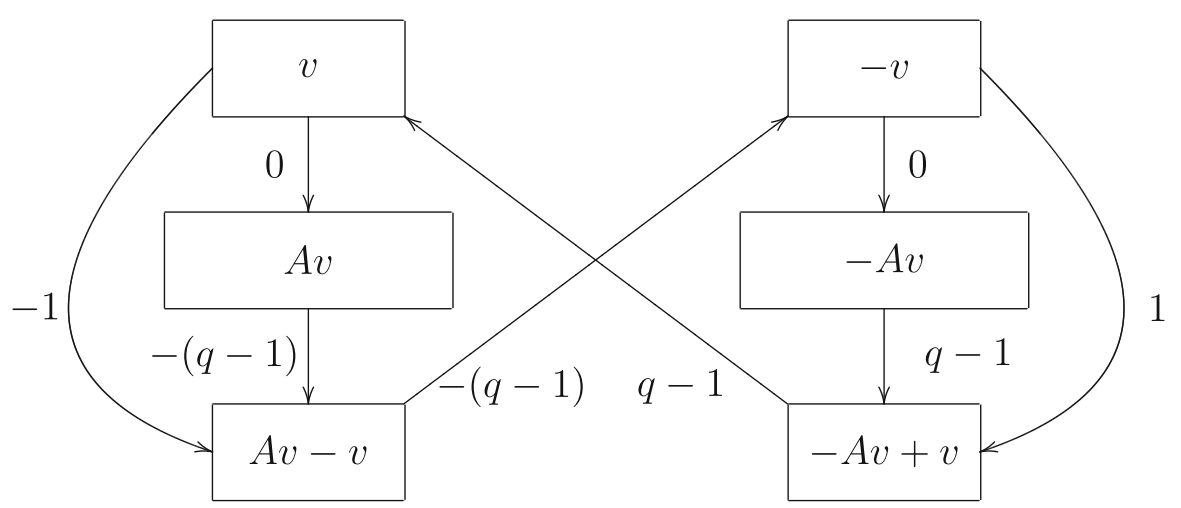

Fig. 5 The neighbor graph of $T$ associated with $f(x)=x^{2}-x+q$

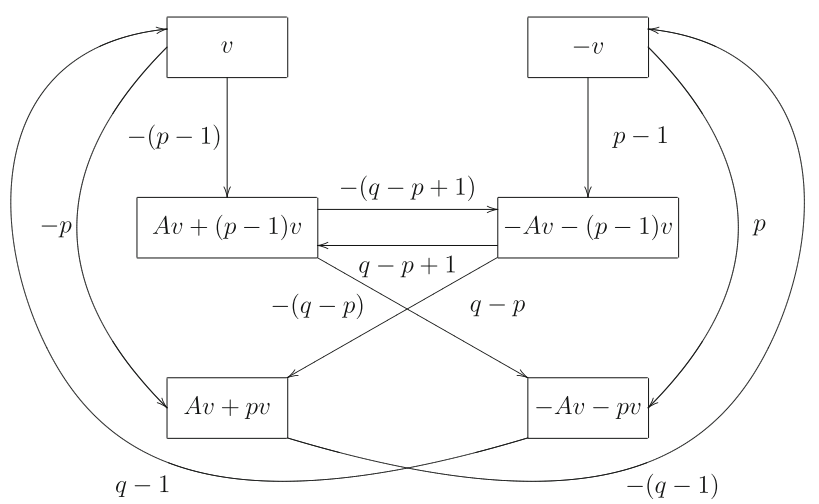

Fig. 6 The neighbor graph of $T$ associated with $f(x)=x^{2}+p x+q, p \geq 2,2 p \leq q+2$ (excluding $q=p=2$ ) 


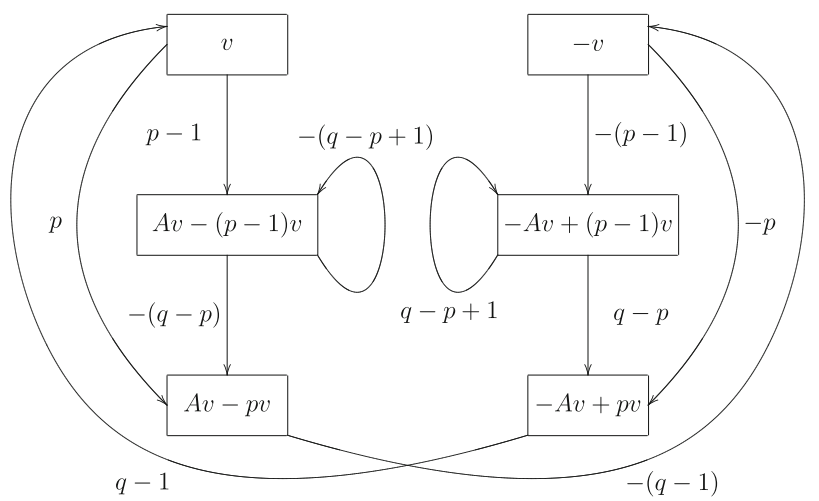

Fig. 7 The neighbor graph of $T$ associated with $f(x)=x^{2}-p x+q, p \geq 2,2 p \leq q+2$ (excluding $q=p=2$ )
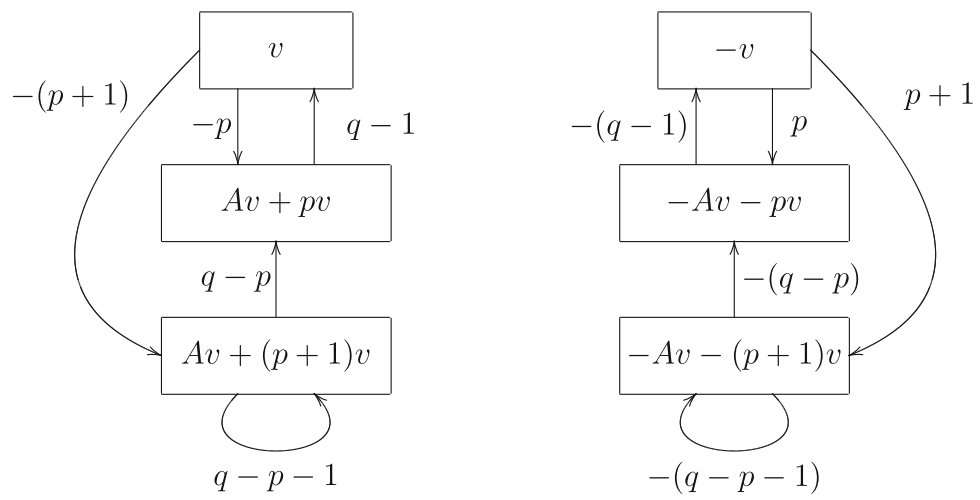

Fig. 8 The neighbor graph of $T$ associated with $f(x)=x^{2}+p x-q, p \geq 1,2 p \leq q-2$

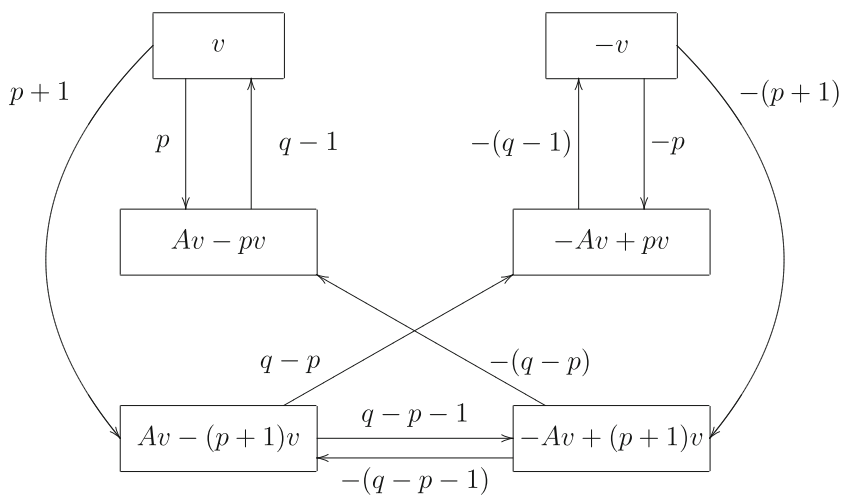

Fig. 9 The neighbor graph of $T$ associated with $f(x)=x^{2}-p x-q, p \geq 1,2 p \leq q-2$ 


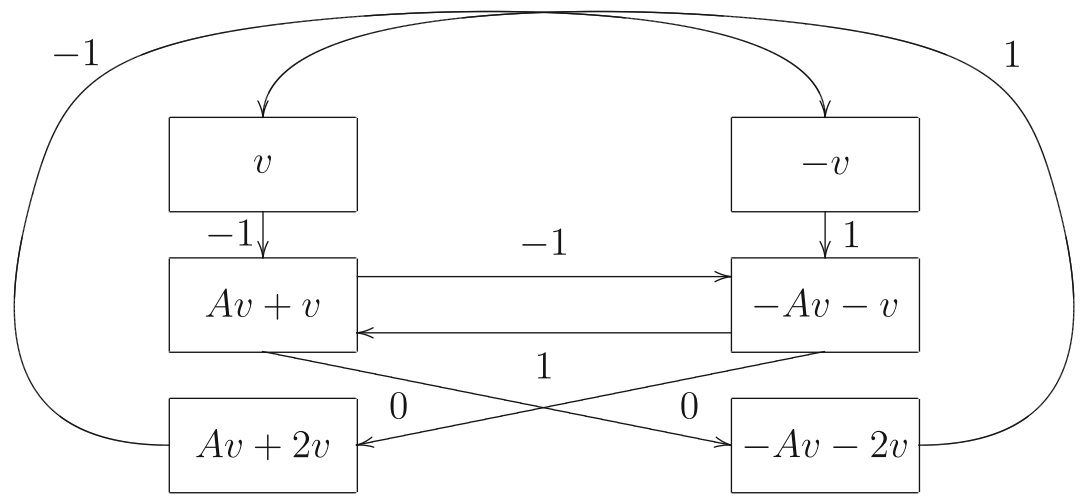

Fig. 10 The neighbor graph of $T$ associated with $f(x)=x^{2}+2 x+2$

Fig. 11 The neighbor graph of $T$ associated with $f(x)=x^{2}-2 x+2$

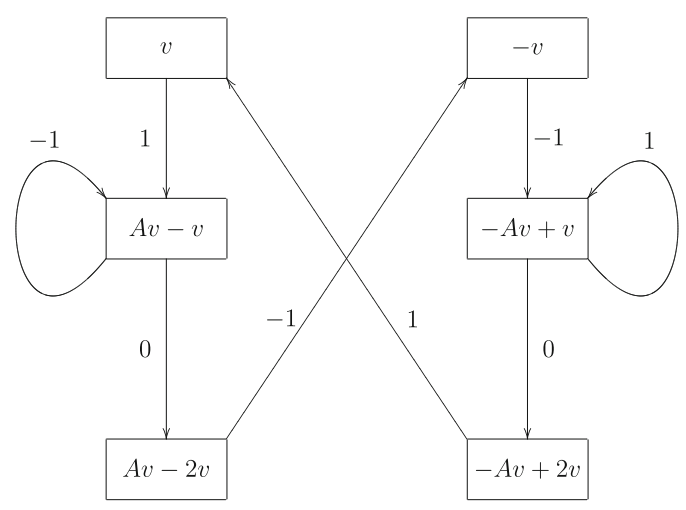

\section{Appendix 2: Graph-Directed Sets}

Let $f(x)=x^{2} \pm p x \pm q(p \geq 0, q \geq 2)$. The graph-directed sets representing the boundary $\partial T$ are classified by $f(x)$ and listed below.

(1) $f(x)=x^{2}+q$. Convention: $u_{1}=v, u_{2}=A v-v, u_{3}=A v, u_{4}=A v+v$.

$$
\begin{aligned}
A T_{u_{1}} & =\bigcup_{j=1}^{q-1}\left(T_{u_{2}}+j v\right) \cup \bigcup_{j=0}^{q-1}\left(T_{u_{3}}+j v\right) \cup \bigcup_{j=0}^{q-2}\left(T_{u_{4}}+j v\right), \\
A T_{u_{2}} & =T_{-u_{4}}, \\
A T_{u_{3}} & =T_{-u_{1}}, \\
A T_{u_{4}} & =T_{u_{2}}, \\
A T_{-u_{1}} & =\bigcup_{j=0}^{q-2}\left(T_{-u_{2}}+j v\right) \cup \bigcup_{j=0}^{q-1}\left(T_{-u_{3}}+j v\right) \cup \bigcup_{j=1}^{q-1}\left(T_{-u_{4}}+j v\right), \\
A T_{-u_{2}} & =T_{u_{4}}+(q-1) v,
\end{aligned}
$$




$$
\begin{aligned}
& A T_{-u_{3}}=T_{u_{1}}+(q-1) v, \\
& A T_{-u_{4}}=T_{-u_{2}}+(q-1) v .
\end{aligned}
$$

(2) $f(x)=x^{2}-q$. Convention: $u_{1}=v, u_{2}=A v-v, u_{3}=A v, u_{4}=A v+v$.

$$
\begin{aligned}
A T_{u_{1}} & =\bigcup_{j=1}^{q-1}\left(T_{u_{2}}+j v\right) \cup \bigcup_{j=0}^{q-1}\left(T_{u_{3}}+j v\right) \cup \bigcup_{j=0}^{q-2}\left(T_{u_{4}}+j v\right), \\
A T_{u_{2}} & =T_{-u_{2}}+(q-1) v, \\
A T_{u_{3}} & =T_{u_{1}}+(q-1) v, \\
A T_{u_{4}} & =T_{u_{4}}+(q-1) v, \\
A T_{-u_{1}} & =\bigcup_{j=0}^{q-2}\left(T_{-u_{2}}+j v\right) \cup \bigcup_{j=0}^{q-1}\left(T_{-u_{3}}+j v\right) \cup \bigcup_{j=1}^{q-1}\left(T_{-u_{4}}+j v\right), \\
A T_{-u_{2}} & =T_{u_{2}}, \\
A T_{-u_{3}} & =T_{-u_{1}}, \\
A T_{-u_{4}} & =T_{-u_{4}} .
\end{aligned}
$$

(3) $f(x)=x^{2}+x+q$. Convention: $u_{1}=v, u_{2}=A v, u_{3}=A v+v$.

$$
\begin{aligned}
A T_{u_{1}} & =\bigcup_{j=0}^{q-1}\left(T_{u_{2}}+j v\right) \cup \bigcup_{j=0}^{q-2}\left(T_{u_{3}}+j v\right), \\
A T_{u_{2}} & =T_{-u_{3}}, \\
A T_{u_{3}} & =T_{-u_{1}}, \\
A T_{-u_{1}} & =\bigcup_{j=0}^{q-1}\left(T_{-u_{2}}+j v\right) \cup \bigcup_{j=1}^{q-1}\left(T_{-u_{3}}+j v\right), \\
A T_{-u_{2}} & =T_{u_{3}}+(q-1) v, \\
A T_{-u_{3}} & =T_{u_{1}}+(q-1) v .
\end{aligned}
$$

(4) $f(x)=x^{2}-x+q$. Convention: $u_{1}=v, u_{2}=A v, u_{3}=A v-v$.

$$
\begin{aligned}
A T_{u_{1}} & =\bigcup_{j=0}^{q-1}\left(T_{u_{2}}+j v\right) \cup \bigcup_{j=0}^{q-2}\left(T_{u_{3}}+j v\right), \\
A T_{u_{2}} & =T_{u_{3}}, \\
A T_{u_{3}} & =T_{-u_{1}}, \\
A T_{-u_{1}} & =\bigcup_{j=0}^{q-1}\left(T_{-u_{2}}+j v\right) \cup \bigcup_{j=1}^{q-1}\left(T_{-u_{3}}+j v\right), \\
A T_{-u_{2}} & =T_{-u_{3}}+(q-1) v, \\
A T_{-u_{3}} & =T_{u_{1}}+(q-1) v .
\end{aligned}
$$


(5) $f(x)=x^{2}+p x+q, p \geq 2,2 p \leq q+2$ (excluding $p=q=2$ ). Convention: $u_{1}=v, u_{2}=A v+(p-1) v, u_{3}=A v+p v$.

$$
\begin{aligned}
A T_{u_{1}} & =\bigcup_{j=0}^{q-p}\left(T_{u_{2}}+j v\right) \cup \bigcup_{j=0}^{q-p-1}\left(T_{u_{3}}+j v\right), \\
A T_{u_{2}} & =\bigcup_{j=0}^{p-2}\left(T_{-u_{2}}+j v\right) \cup \bigcup_{j=0}^{p-1}\left(T_{-u_{3}}+j v\right), \\
A T_{u_{3}} & =T_{-u_{1}}, \\
A T_{-u_{1}} & =\bigcup_{j=p-1}^{q-1}\left(T_{-u_{2}}+j v\right) \cup \bigcup_{j=p}^{q-1}\left(T_{-u_{3}}+j v\right), \\
A T_{-u_{2}} & =\bigcup_{j=q-p+1}^{q-1}\left(T_{u_{2}}+j v\right) \cup \bigcup_{j=q-p}^{q-1}\left(T_{u_{3}}+j v\right), \\
A T_{-u_{3}} & =T_{u_{1}}+(q-1) v .
\end{aligned}
$$

(6) $f(x)=x^{2}-p x+q, p \geq 2,2 p \leq q+2$ (excluding $p=q=2$ ). Convention: $u_{1}=v, u_{2}=A v-(p-1) v, u_{3}=A v-p v$.

$$
\begin{aligned}
A T_{u_{1}} & =\bigcup_{j=p-1}^{q-1}\left(T_{u_{2}}+j v\right) \cup \bigcup_{j=p}^{q-1}\left(T_{u_{3}}+j v\right), \\
A T_{u_{2}} & =\bigcup_{j=0}^{p}\left(T_{u_{2}}+j v\right) \cup \bigcup_{j=0}^{p-1}\left(T_{u_{3}}+j v\right), \\
A T_{u_{3}} & =T_{-u_{1}}, \\
A T_{-u_{1}} & =\bigcup_{j=0}^{q-p}\left(T_{-u_{2}}+j v\right) \cup \bigcup_{j=0}^{q-p-1}\left(T_{-u_{3}}+j v\right), \\
A T_{-u_{2}} & =\bigcup_{j=q-p+1}^{q-1}\left(T_{-u_{2}}+j v\right) \cup \bigcup_{j=q-p}^{q-1}\left(T_{-u_{3}}+j v\right), \\
A T_{-u_{3}}= & T_{u_{1}}+(q-1) v .
\end{aligned}
$$

(7) $f(x)=x^{2}+p x-q, p \geq 1,2 p \leq q-2$. Convention: $u_{1}=v, u_{2}=A v+$ $p v, u_{3}=A v+(p+1) v$.

$$
\begin{aligned}
& A T_{u_{1}}=\bigcup_{j=0}^{q-p-1}\left(T_{u_{2}}+j v\right) \cup \bigcup_{j=0}^{q-p-2}\left(T_{u_{3}}+j v\right), \\
& A T_{u_{2}}=T_{u_{1}}+(q-1) v,
\end{aligned}
$$




$$
\begin{aligned}
A T_{u_{3}} & =\bigcup_{j=q-p}^{q-1}\left(T_{u_{2}}+j v\right) \cup \bigcup_{j=q-p-1}^{q-1}\left(T_{u_{3}}+j v\right), \\
A T_{-u_{1}}= & \bigcup_{j=p}^{q-1}\left(T_{-u_{2}}+j v\right) \cup \bigcup_{j=p+1}^{q-1}\left(T_{-u_{3}}+j v\right), \\
A T_{-u_{2}}= & T_{u_{1}}, \\
A T_{-u_{3}}= & \bigcup_{j=0}^{p-1}\left(T_{-u_{2}}+j v\right) \cup \bigcup_{j=0}^{p}\left(T_{-u_{3}}+j v\right) .
\end{aligned}
$$

(8) $f(x)=x^{2}-p x-q, p \geq 1,2 p \leq q-2$. Convention: $u_{1}=v, u_{2}=A v-$ $p v, u_{3}=A v-(p+1) v$.

$$
\begin{aligned}
A T_{u_{1}}= & \bigcup_{j=p}^{q-1}\left(T_{u_{2}}+j v\right) \cup \bigcup_{j=p+1}^{q-1}\left(T_{u_{3}}+j v\right), \\
A T_{u_{2}}= & T_{u_{1}}+(q-1) v, \\
A T_{u_{3}}= & \bigcup_{j=q-p}^{q-1}\left(T_{-u_{2}}+j v\right) \cup \bigcup_{j=q-p-1}^{q-1}\left(T_{-u_{3}}+j v\right), \\
A T_{-u_{1}}= & \bigcup_{j=0}^{q-p-1}\left(T_{-u_{2}}+j v\right) \cup \bigcup_{j=0}^{q-p-2}\left(T_{-u_{3}}+j v\right), \\
A T_{-u_{2}}= & T_{-u_{1}}, \\
A T_{-u_{3}}= & \bigcup_{j=0}^{p-1}\left(T_{u_{2}}+j v\right) \cup \bigcup_{j=0}^{p-2}\left(T_{u_{3}}+j v\right) .
\end{aligned}
$$

(9) $f(x)=x^{2}+2 x+2$. Convention: $u_{1}=v, u_{2}=A v+v, u_{3}=A v+2 v$.

$$
\begin{aligned}
A T_{u_{1}} & =T_{u_{2}}, \\
A T_{u_{2}} & =T_{-u_{2}} \cup T_{-u_{3}} \cup\left(T_{-u_{3}}+v\right), \\
A T_{u_{3}} & =T_{-u_{1}} \\
A T_{-u_{1}} & =T_{-u_{2}}+v, \\
A T_{-u_{2}} & =\left(T_{u_{2}}+v\right) \cup T_{u_{3}} \cup\left(T_{u_{3}}+v\right), \\
A T_{-u_{3}} & =T_{u_{1}}+v
\end{aligned}
$$

(10) $f(x)=x^{2}-2 x+2$. Convention: $u_{1}=v, u_{2}=A v-v, u_{3}=A v-2 v$.

$$
\begin{aligned}
& A T_{u_{1}}=T_{u_{2}}+v, \\
& A T_{u_{2}}=T_{u_{2}} \cup T_{u_{3}} \cup\left(T_{u_{3}}+v\right), \\
& A T_{u_{3}}=T_{-u_{1}},
\end{aligned}
$$




$$
\begin{aligned}
& A T_{-u_{1}}=T_{-u_{2}}, \\
& A T_{-u_{2}}=\left(T_{-u_{2}}+v\right) \cup T_{-u_{3}} \cup\left(T_{-u_{3}}+v\right), \\
& A T_{-u_{3}}=T_{u_{1}}+v .
\end{aligned}
$$

\section{Appendix 3: Contact Matrices}

Let $f(x)=x^{2} \pm p x \pm q(p \geq 0, q \geq 2)$. The contact matrices (in table form) of disk-like tiles are classified by $f(x)$ and listed in Tables 4, 5, 6, 7, 8, 9, 10, 11, 12, and 13.

Table $4 f(x)=x^{2}+q$

\begin{tabular}{lllllllll}
\hline & $v$ & $A v$ & $-v$ & $-A v$ & $A v-v$ & $-A v-v$ & $-A v+v$ & $A v+v$ \\
\hline$v$ & 0 & $q$ & 0 & 0 & $q-1$ & 0 & 0 & $q-1$ \\
$A v$ & 0 & 0 & 1 & 0 & 0 & 0 & 0 & 0 \\
$-v$ & 0 & 0 & 0 & $q$ & 0 & $q-1$ & $q-1$ & 0 \\
$-A v$ & 1 & 0 & 0 & 0 & 0 & 0 & 0 & 0 \\
$A v-v$ & 0 & 0 & 0 & 0 & 0 & 1 & 0 & 0 \\
$-A v-v$ & 0 & 0 & 0 & 0 & 0 & 0 & 1 & 0 \\
$-A v+v$ & 0 & 0 & 0 & 0 & 0 & 0 & 0 & 1 \\
$A v+v$ & 0 & 0 & 0 & 0 & 1 & 0 & 0 & 0 \\
\hline
\end{tabular}

Table $5 f(x)=x^{2}-q$

\begin{tabular}{lllllllll}
\hline & $v$ & $A v$ & $-v$ & $-A v$ & $A v-v$ & $-A v+v$ & $A v+v$ & $-A v-v$ \\
\hline$v$ & 0 & $q$ & 0 & 0 & $q-1$ & 0 & $q-1$ & 0 \\
$A v$ & 1 & 0 & 0 & 0 & 0 & 0 & 0 & 0 \\
$-v$ & 0 & 0 & 0 & $q$ & 0 & $q-1$ & 0 & $q-1$ \\
$-A v$ & 0 & 0 & 1 & 0 & 0 & 0 & 0 & 0 \\
$A v-v$ & 0 & 0 & 0 & 0 & 0 & 1 & 0 & 0 \\
$-A v+v$ & 0 & 0 & 0 & 0 & 1 & 0 & 0 & 0 \\
$A v+v$ & 0 & 0 & 0 & 0 & 0 & 0 & 1 & 0 \\
$-A v-v$ & 0 & 0 & 0 & 0 & 0 & 0 & 0 & 1 \\
\hline
\end{tabular}

Table $6 f(x)=x^{2}+x+q$

\begin{tabular}{lllllll}
\hline & $v$ & $A v$ & $A v+v$ & $-v$ & $-A v$ & $-A v-v$ \\
\hline$v$ & 0 & $q$ & $q-1$ & 0 & 0 & 0 \\
$A v$ & 0 & 0 & 0 & 0 & 0 & 1 \\
$A v+v$ & 0 & 0 & 0 & 1 & 0 & 0 \\
$-v$ & 0 & 0 & 0 & 0 & $q$ & $q-1$ \\
$-A v$ & 0 & 0 & 1 & 0 & 0 & 0 \\
$-A v-v$ & 1 & 0 & 0 & 0 & 0 & 0 \\
\hline
\end{tabular}


Table $7 f(x)=x^{2}-x+q$

\begin{tabular}{lllllll}
\hline & $v$ & $A v$ & $A v-v$ & $-v$ & $-A v$ & $-A v+v$ \\
\hline$v$ & 0 & $q$ & $q-1$ & 0 & 0 & 0 \\
$A v$ & 0 & 0 & 1 & 0 & 0 & 0 \\
$A v-v$ & 0 & 0 & 0 & 1 & 0 & 0 \\
$-v$ & 0 & 0 & 0 & 0 & $q$ & $q-1$ \\
$-A v$ & 0 & 0 & 0 & 0 & 0 & 1 \\
$-A v+v$ & 1 & 0 & 0 & 0 & 0 & 0 \\
\hline
\end{tabular}

Table $8 f(x)=x^{2}+p x+q, p \geq 2,2 p \leq q+2$ (excluding $p=q=2$ )

\begin{tabular}{lllllll}
\hline & $v$ & $A v+(p-1) v$ & $A v+p v$ & $-v$ & $-A v-(p-1) v$ & $-A v-p v$ \\
\hline$v$ & 0 & $q-p+1$ & $q-p$ & 0 & 0 & 0 \\
$A v+(p-1) v$ & 0 & 0 & 0 & 0 & $p-1$ & $p$ \\
$A v+p v$ & 0 & 0 & 0 & 1 & 0 & 0 \\
$-v$ & 0 & 0 & 0 & 0 & $q-p+1$ & $q-p$ \\
$-A v-(p-1) v$ & 0 & $p-1$ & $p$ & 0 & 0 & 0 \\
$-A v-p v$ & 1 & 0 & 0 & 0 & 0 & 0 \\
\hline
\end{tabular}

Table $9 f(x)=x^{2}-p x+q, p \geq 2,2 p \leq q+2($ excluding $p=q=2$ )

\begin{tabular}{lllllll}
\hline & $v$ & $A v-(p-1) v$ & $A v-p v$ & $-v$ & $-A v+(p-1) v$ & $-A v+p v$ \\
\hline$v$ & 0 & $q-p+1$ & $q-p$ & 0 & 0 & 0 \\
$A v-(p-1) v$ & 0 & $p-1$ & $p$ & 0 & 0 & 0 \\
$A v-p v$ & 0 & 0 & 0 & 1 & 0 & 0 \\
$-v$ & 0 & 0 & 0 & 0 & $q-p+1$ & $q-p$ \\
$-A v+(p-1) v$ & 0 & 0 & 0 & 0 & $p-1$ & $p$ \\
$-A v+p v$ & 1 & 0 & 0 & 0 & 0 & 0 \\
\hline
\end{tabular}

Table $10 f(x)=x^{2}+p x-q, p \geq 1,2 p \leq q-2$

\begin{tabular}{lllllll}
\hline & $v$ & $A v+p v$ & $A v+(p+1) v$ & $-v$ & $-A v-p v$ & $-A v-(p+1) v$ \\
\hline$v$ & 0 & $q-p$ & $q-p-1$ & 0 & 0 & 0 \\
$A v+p v$ & 1 & 0 & 0 & 0 & 0 & 0 \\
$A v+(p+1) v$ & 0 & $p$ & $p+1$ & 0 & 0 & 0 \\
$-v$ & 0 & 0 & 0 & 0 & $q-p$ & $q-p-1$ \\
$-A v-p v$ & 0 & 0 & 0 & 1 & 0 & 0 \\
$-A v-(p+1) v$ & 0 & 0 & 0 & 0 & $p$ & $p+1$ \\
\hline
\end{tabular}


Table $11 f(x)=x^{2}-p x-q, p \geq 1,2 p \leq q-2$

\begin{tabular}{lllllll}
\hline & $v$ & $A v-p v$ & $A v-(p+1) v$ & $-v$ & $-A v+p v$ & $-A v+(p+1) v$ \\
\hline$v$ & 0 & $q-p$ & $q-p-1$ & 0 & 0 & 0 \\
$A v-p v$ & 1 & 0 & 0 & 0 & 0 & 0 \\
$A v-(p+1) v$ & 0 & 0 & 0 & 0 & $p$ & $p+1$ \\
$-v$ & 0 & 0 & 0 & 0 & $q-p$ & $q-p-1$ \\
$-A v+p v$ & 0 & 0 & 0 & 1 & 0 & 0 \\
$-A v+(p+1) v$ & 0 & $p$ & $p+1$ & 0 & 0 & 0 \\
\hline
\end{tabular}

Table $12 f(x)=x^{2}+2 x+2$

\begin{tabular}{lllllll}
\hline & $v$ & $A v+v$ & $A v+2 v$ & $-v$ & $-A v-v$ & $-A v-2 v$ \\
\hline$v$ & 0 & 1 & 0 & 0 & 0 & 0 \\
$A v+v$ & 0 & 0 & 0 & 0 & 1 & 2 \\
$A v+2 v$ & 0 & 0 & 0 & 1 & 0 & 0 \\
$-v$ & 0 & 0 & 0 & 0 & 1 & 0 \\
$-A v-v$ & 0 & 1 & 2 & 0 & 0 & 0 \\
$-A v-2 v$ & 1 & 0 & 0 & 0 & 0 & 0 \\
\hline
\end{tabular}

Table $13 f(x)=x^{2}-2 x+2$

\begin{tabular}{lllllll}
\hline & $v$ & $A v-v$ & $A v-2 v$ & $-v$ & $-A v+v$ & $-A v+2 v$ \\
\hline$v$ & 0 & 1 & 0 & 0 & 0 & 0 \\
$A v-v$ & 0 & 1 & 2 & 0 & 0 & 0 \\
$A v-2 v$ & 0 & 0 & 0 & 1 & 0 & 0 \\
$-v$ & 0 & 0 & 0 & 0 & 1 & 0 \\
$-A v+v$ & 0 & 0 & 0 & 0 & 1 & 2 \\
$-A v+2 v$ & 1 & 0 & 0 & 0 & 0 & 0 \\
\hline
\end{tabular}

\section{References}

1. Akiyama, S., Loridant, B.: Boundary parametrization of planar self-affine tiles with collinear digit set. Sci. China Math. 53(9), 2173-2194 (2010)

2. Akiyama, S., Loridant, B.: Boundary parametrization of self-affine tiles. J. Math. Soc. Jpn. 63(2), 525-579 (2011)

3. Bandt, C., Gelbrich, G.: Classification of self-affine lattice tilings. J. Lond. Math. Soc. 50, 581-593 (1994)

4. Bandt, C., Mesing, M.: Self-affine fractals of finite type. Banach Center Publ. 84, 131-148 (2009)

5. Bandt, C., Wang, Y.: Disk-like self-affine tiles in $\mathbb{R}^{2}$. Discrete Comput. Geom. 26, 591-601 (2001)

6. Deng, Q.R., Lau, K.S.: Connectedness of a class of planar self-affine tiles. J. Math. Anal. Appl. 380, 493-500 (2011)

7. Deng, D.-W., Jiang, T., Ngai, S.-M.: Structure of planar integral self-affine tilings. Math. Nachr. 285, $447-475$ (2012) 
8. Duval, P., Keesling, J., Vince, A.: The Hausdorff dimension of the boundary of a self-similar tile. J. Lond. Math. Soc. 61, 748-760 (2000)

9. Falconer, K.J.: Techniques in Fractal Geometry. Wiley, New York (1997)

10. Falconer, K.J.: Fractal Geometry: Mathematical Foundations and Applications. Wiley, New York (2003)

11. Fischer, R.: Sofic systems and graphs. Monatsh. Math. 80, 179-186 (1975)

12. Gantmacher, F.R.: Matrix Theory, vol. II. Chelsea Publishing Company, New York (1960)

13. Gilbert, W.G.: Radix representataions of quadratic fields. J. Math. Anal. Appl. 83, 264-274 (1981)

14. Gmainer, J., Thuswaldner, J.M.: On disk-like self-affine tiles arising from polyominoes. Methods Appl. Anal. 13(4), 351-372 (2006)

15. Gröchenig, K., Haas, A.: Self-similar lattice tilings. J. Fourier Anal. Appl. 1, 131-170 (1994)

16. He, X.G., Lau, K.S.: On a generalized dimension of self-affine fractals. Math. Nachr. 281(8), 11421158 (2008)

17. He, X.G., Lau, K.S., Rao, H.: Self-affine sets and graph-directed systems. Constr. Approx. 19, 373-397 (2003)

18. Kirat, I.: Disk-like tiles and self-affine curves with non-collinear digits. Math. Comput. 79, 1019-1045 (2010)

19. Kirat, I., Lau, K.S.: On the connectedness of self-affine tiles. J. Lond. Math. Soc. 62, 291-304 (2000)

20. Lagarias, J.C., Wang, Y.: Self-affine tiles in $\mathbb{R}^{n}$. Adv. Math. 121, 21-49 (1996)

21. Lagarias, J.C., Wang, Y.: Integral Self-affine tiles in $\mathbb{R}^{n}$. I. Standard and non-standard digit sets. J. Lond. Math. Soc. 54, 161-179 (1996)

22. Lagarias, J.C., Wang, Y.: Integral self-affine tiles in $\mathbb{R}^{n}$. II. Lattice tilings. J. Fourier Anal. Appl. 3, 84-102 (1997)

23. Lau, K.S., Ngai, S.M.: Dimensions of the boundaries of self-similar sets. Exp. Math. 12, 13-26 (2003)

24. Leung, K.S., Lau, K.S.: Disk-likeness of planar self-affine tiles. Trans. Am. Math. Soc. 359, 3337-3355 (2007)

25. Leung, K.S., Luo, J.J.: Connectedness of planar self-affine sets associated with non-consecutive collinear digit sets. J. Math. Anal. Appl. 395, 208-217 (2012)

26. Luo, J.J., Yang, Y.M.: On single-matrix graph-directed iterated function systems. J. Math. Anal. Appl. 372, 8-18 (2010)

27. Lind, D., Marcus, B.: An Introduction to Symbolic Dynamics and Coding. Cambridge University Press, Cambridge (1995)

28. Mauldin, R.D., Williams, S.C.: Hausdorff dimension in graph-directed constructions. Trans. Am. Math. Soc. 309, 811-829 (1988)

29. Müller, W., Thuswaldner, J.M., Tichy, R.T.: Fractal properties of number system. Period. Math. Hungar. 42, 51-68 (2001)

30. Seneta, E.: Non-negative Matrices and Markov Chains, 2nd edn. Springer, New York (1980)

31. Strichartz, R.S., Wang, Y.: Geometry of self-affine tiles. I. Indiana Univ. Math. J. 48, 1-23 (1999)

32. Veerman, J.: Hausdorff dimension of boundaries of self-affine tiles in $\mathbb{R}^{n}$. Bol. Soc. Mat. Mexicana III 4(2), 159C182 (1998)

33. Weiss, B.: Subshifts of finite type and sofic systems. Monatsh. Math. 77, 462-474 (1973) 